\title{
AFP: First Experience with Data and Future Plans
}

\author{
Grzegorz Gach, on behalf of the ATLAS Collaboration \\ AGH University of Science and Technology, Cracow, Poland \\ E-mail: grzegorz.gachefis.agh.edu.pl
}

\begin{abstract}
The ATLAS Forward Proton (AFP) detector system aims at the measurement of protons scattered diffractively or electromagnetically at very small angles. The first arm of the system was installed in 2016 and AFP took data in several commissioning and physics runs. The installation of the second arm is ongoing and will be completed in time for the 2017 data taking period. This will allow the measurements of processes with two tagged forward protons being a signature of: central diffraction, exclusive production, and two-photon processes. The early results and experience from the first year of data taking is presented together with the status of the secondarm installation and plans for the future.
\end{abstract}

XXV International Workshop on Deep-Inelastic Scattering and Related Subjects

3-7 April 2017

University of Birmingham, $U K$ 


\section{ATLAS and Forward Detectors}

The main ATLAS [1] apparatus is a general-purpose detector operating at the LHC [2] accelerator. It is capable of detecting products of collisions in a full range of the azimuthal angle and in pseudorapidity ${ }^{1}$ roughly between -5 and 5 . Particles that are produced at small angles escape undetected from the main detector. In order to enable measurements of at least part of the final state propagating close to or inside the beam pipe a set of forward detectors was designed and installed.

Currently, there are four forward detectors. The closest to the nominal interaction point, IP, is LUCID, which is placed $17 \mathrm{~m}$ away from it. The main purpose of this detector is the luminosity measurement. The next detector is placed $140 \mathrm{~m}$ from the IP and is called the Zero Degree Calorimeter, ZDC. Its aim is to measure neutral particles produced at very small angles, predominantly neutrons. The furthest detector is the Absolute Luminosity For ATLAS, ALFA [3], and is installed about $240 \mathrm{~m}$ from the IP. The newest detector the ATLAS Forward Proton, AFP [4], is presented in this article.

\section{ATLAS Forward Proton Detector}

The AFP detector is dedicated to the measurement of diffractively scattered protons and consists of four roman pot stations. On each side of the main ATLAS detector two of them are placed. The near stations are located $205 \mathrm{~m}$ away from the IP, while the far stations are $12 \mathrm{~m}$ further. The roman pots allow horizontal movement of the detectors and positioning them very close to the beam. This enables the measurement of protons with relative energy loss, $\xi=\left(E-E^{\prime}\right) / E$, within the range $(0.025,0.1)$.

Each station is equipped with a tracking detector [5, 6]. It consists of four layers of silicon 3D pixels. Each layer is build of $336 \times 80$ pixels each of size $50 \times 250 \mu \mathrm{m}^{2}$. The assembled layers are presented in the left photograph in Figure 1. The pixel modules technology is well known and tested, because the same modules are used in ATLAS Insertable B-Layer [7]. The detectors are horizontally rotated by $14^{\circ}$. A diffractive proton passing a layer positioned in this way is expected to hit two pixels. Information about fired pixels is used to reconstruct position and direction of the proton trajectory. This knowledge combined with information about settings of the LHC magnets allows reconstruction of the proton momentum.

Far stations additionally house the time-of-flight counters [6, 8]. Time measurement is done using the so-called "trains" i.e. sets of four quartz LQBars. A photograph of two such trains is presented on the right hand-side of Figure 1. All bars measure the arrival time of the same proton. Calculation of the mean value of the four measurements improves time evaluation. In each far station four trains are mounted. The granularity provided by four trains should be sufficient to measure one proton in one train in most of the events. The designed time resolution is $10 \mathrm{ps}$. This translates to the resolution of the reconstruction of the vertex $Z$ coordinate of about $\sigma_{z}=$ $2.1 \mathrm{~mm}$. The designed detector efficiency is greater than $90 \%$. Additionally, the counters are fast enough to provide the trigger signal. This is crucial for collecting data with a high average number of interactions per bunch crossing, because it allows matching of the protons in AFP with the reconstructed vertex and selection of the events based on kinematics. This strategy significantly

\footnotetext{
${ }^{1} \eta=-\ln (\tan (\theta / 2))$, where $\theta$ is polar angle
} 
reduces the background originating from pile-up i.e. other interactions occurring in the same bunch crossing.
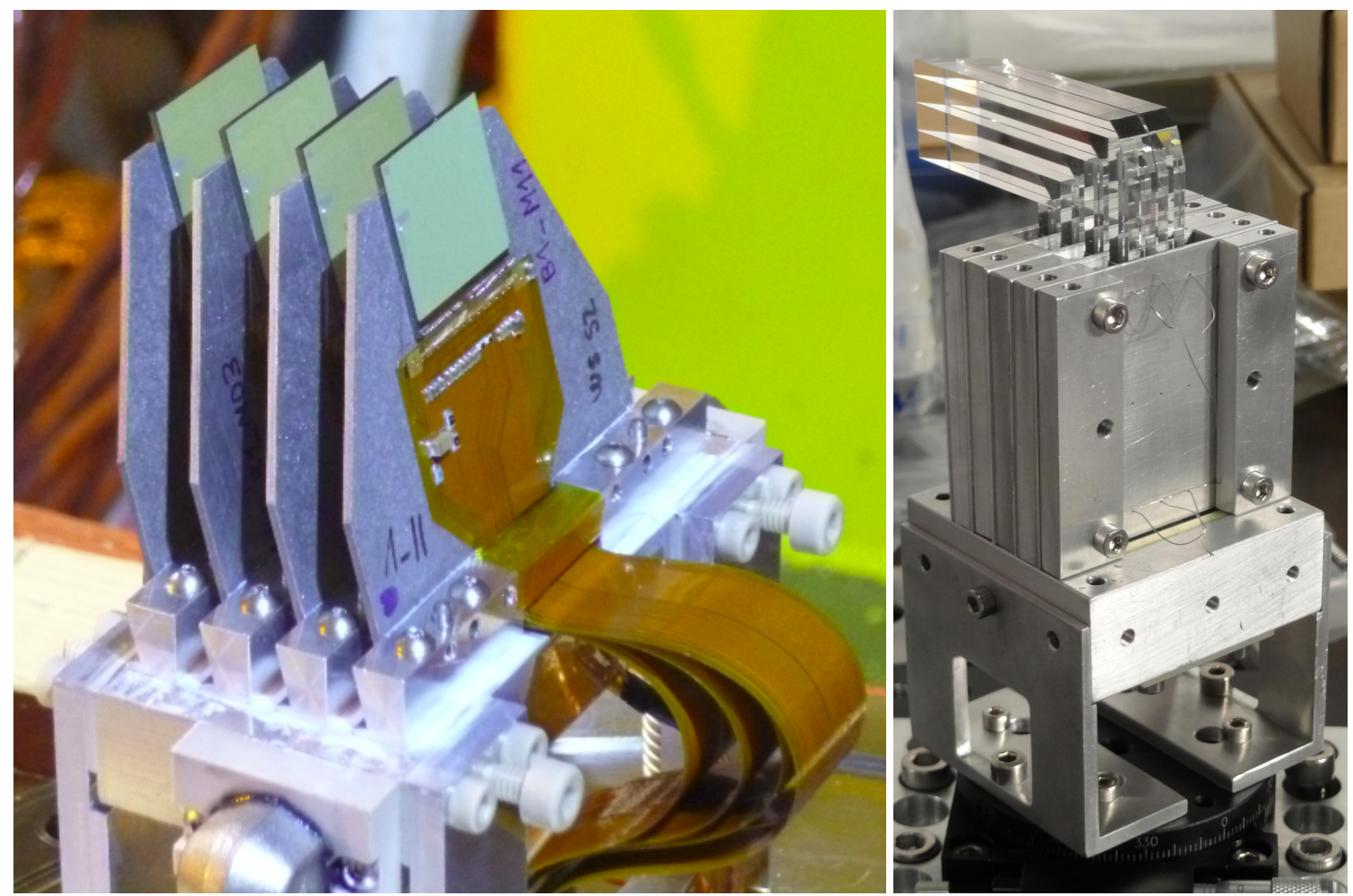

Figure 1: Left: four layers of the assembled silicon pixel detectors. Right: two trains of the time-of-flight counters in the test stand.

Installation of the AFP detector was planned in two stages. The first arm was installed in 2016 and the second in 2017. Both in time for data taking of the LHC collisions.

\section{AFP in 2016}

In 2016 two stations on one side of the main ATLAS detector were installed. Each station was equipped with tracking detectors. The installation was followed by the LHC qualification which is required since the detectors are inserted into the beam pipe. The AFP data acquisition and trigger systems were successfully integrated with their counterparts in the central ATLAS system.

Operation in 2016 provided data from many commissioning runs with various positions of the detectors. There were also two runs dedicated to the AFP physics with the average number of interactions per bunch crossing $\mu \approx 0.03$ and $\mu \approx 0.3$. In total the integrated luminosity of about $500 \mathrm{nb}^{-1}$ was collected.

The first data confirm good performance of the detectors. The left panel of Figure 2 shows a map of tracks in the AFP obtained using single diffractive Monte Carlo, while on the right-hand side a measured map of the pixel hits is shown. In both plots a distinct pattern of diffractive protons is visible.

Good performance of the tracking detectors is also confirmed by the data presented in Figure 3. The left panel presents a number of the pixel hits per event in a plane is presented. As expected 

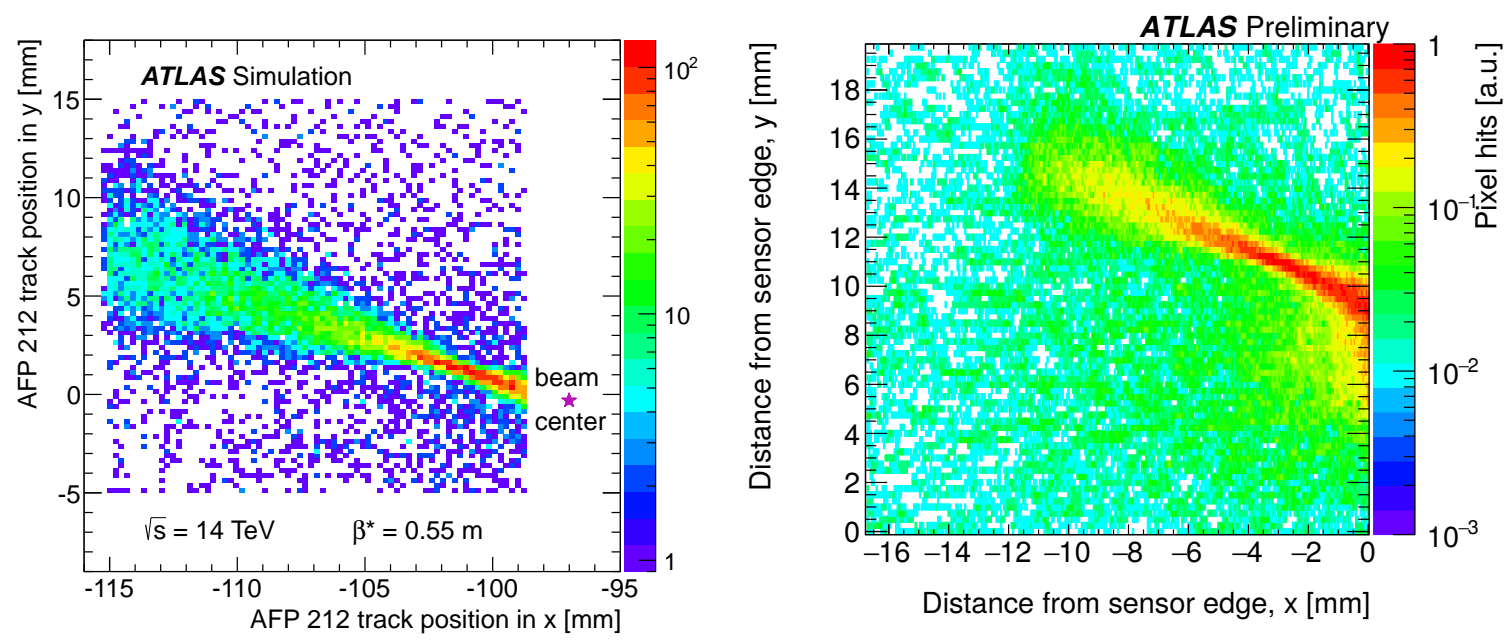

Figure 2: Left: a simulated map of tracks in the AFP far station placed at $212 \mathrm{~m}$ away from the IP. Right: a map of the measured hits in the first layer of the near station [9].

from the detector planes tilt, the majority of the events had signal in two pixels. On the right-hand side distribution the correlation between pixels rows in the first and second planes is presented. In order to reduce the number of possible combinations only events with up to two pixels fired in each plane were considered. A strong correlation is observed, which indicates that the signal originates from passing particles.

Natural physics program for data collected in 2016 consists of measurements of single diffraction. These processes are characterised by the presence of a proton well separated in rapidity from a dissociated system in the final state. Such a proton, produced at small angle, can be measured by the AFP detector. A diagram of such a process is presented in the left panel of Figure 4. The collected integrated luminosity should be sufficient for studies of such processes, because their cross-section is relatively high.

Reconstruction of the proton momentum gives access to the quantities that were not measured directly so far by ATLAS, like relative proton energy loss, $\xi$, or Mandelstam variable $t=\left(\mathbf{P}-\mathbf{P}^{\prime}\right)^{2}$, where $\mathbf{P}$ and $\mathbf{P}^{\prime}$ are initial and final state proton four-momenta, respectively. Currently, two analyses using 2016 data are ongoing. The first one is focused on inclusive single diffractive dissociation and aims at measuring general properties of this process. The second analysis studies diffractive dijet production. Results of the latter can be compared to perturbative QCD calculations. This is possible due to the presence of a hard scale in form of the jets transverse momentum.

\section{4. $\mathbf{A F P}$ in 2017}

AFP installation will be completed in 2017. The remaining two stations are already installed. The tracking detectors will be installed in the new stations and will be replaced in the old stations. The time-of-flight counters will be installed in far stations. Additionally, they have to be integrated with the ATLAS central trigger system. The whole system will have to pass the LHC qualification again. In 2017 there are plans to collect data in special runs with a small number of interactions per bunch crossing and also in standard runs with high pile-up. The presence of AFP on both 

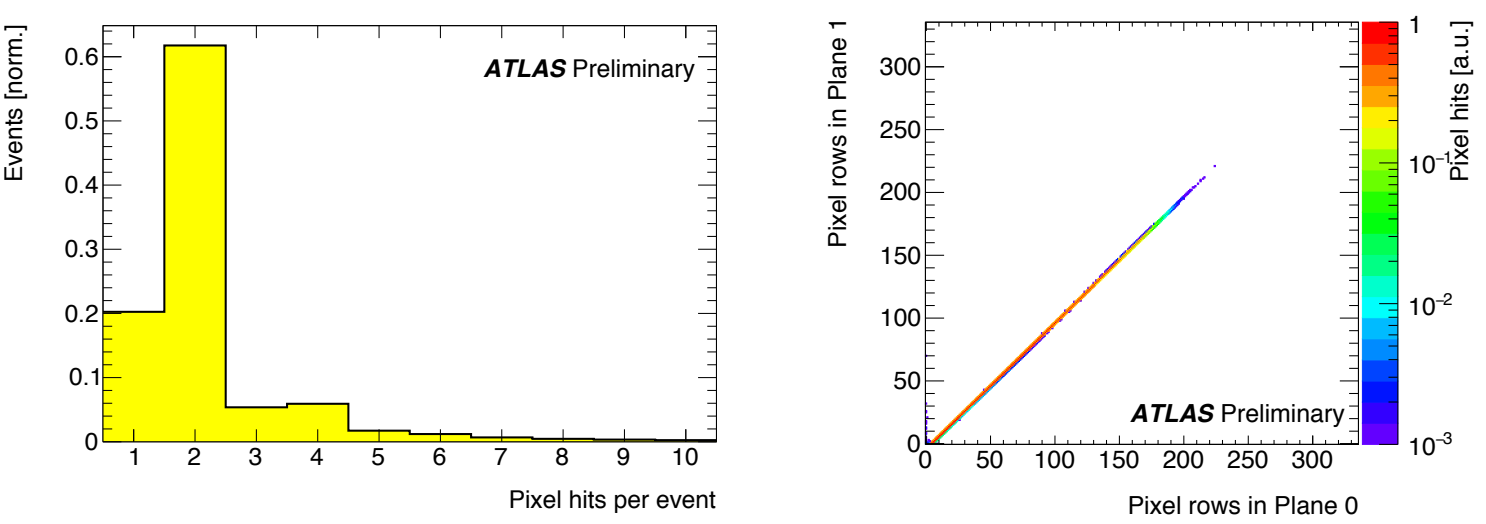

Figure 3: Left: a distribution of number of pixels fired in a pixel plane per event. Right: a distribution of the fired pixel rows in the second plane as a function of row in the first plane. Only events with no more than two hits per plane were considered [9].

sides of the main detector will give a possibility to directly measure central diffractive processes, which final state consists of two protons well separated in rapidity from the dissociated system. The general analysis strategy will be to reconstruct the scattered protons momenta using the AFP and dissociated system with the central detector. This should provide full information about the event kinematics.

The special runs will provide data for studies of single diffraction and central diffractive processes with relatively high cross-sections. The advantage of these runs will be a very clean environment, which should allow precise measurements. One of the planned analyses is study of jet production in central diffractive events.

The standard runs will provide access to the processes with two forward protons in the final state and having small cross-sections. Into this category fall: central diffraction, exclusive and photon induced processes. Several analyses are already planned with 2017 data. One of the most challenging is the measurement of exclusive dijet production. The process is very interesting from the physics point of view, because it can be calculated within QCD without the Pomeron concept, but because of very small cross-section and many background sources it is very difficult to measure. Another interesting group of measurements are the photon induced processes. Access to them should be much easier, because tagging of the forward proton significantly suppresses the backgrounds.

\section{Summary}

The installation of ATLAS Forward Proton detectors, dedicated to measurements of diffractive protons, consists of two stages. The first one was completed in 2016 and allowed for collecting data with measurement of the proton on only one side of the central detector. About $500 \mathrm{nb}^{-1}$ of data for physics analysis was collected for studies of single diffraction. The data is already being analysed.

The final installation stage will be completed during 2017. Time-of-flight counters will allow proton measurements in standard runs at high pile-up providing access to processes with small 

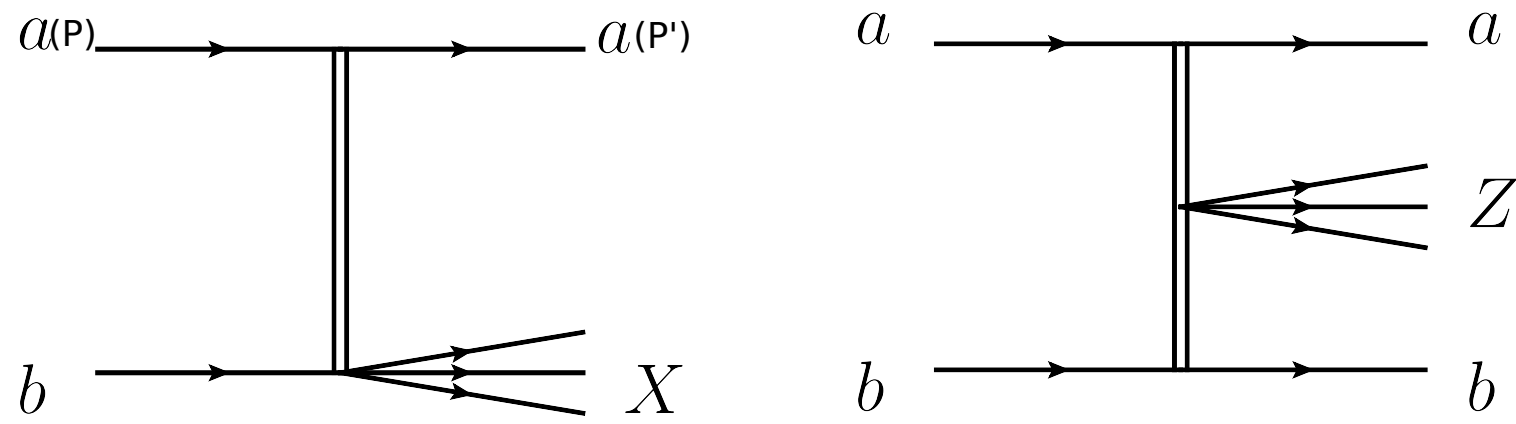

Figure 4: Diffractive processes of main interest to analyses with the AFP detector: single diffraction (left) and central diffraction (right).

cross-sections. Analyses are planned with new data focusing mainly on central diffraction and exclusive processes, in addition to studies of single diffraction.

\section{Acknowledgements}

This work was partly supported by the National Science Centre of Poland under contract No. UMO-2015/19/B/ST2/00989

\section{References}

[1] ATLAS Collaboration, The ATLAS Experiment at the CERN Large Hadron Collider, JINST 3 (2008) S08003.

[2] L. Evans and P. Bryant, LHC Machine, JINST 3 (2008) S08001.

[3] S. Abdel Khalek et al., The ALFA Roman Pot Detectors of ATLAS, JINST 11 (2016) P11013, [1609.00249].

[4] ATLAS Collaboration, Technical Design Report for the ATLAS Forward Proton Detector, Tech. Rep. CERN-LHCC-2015-009. ATLAS-TDR-024, May, 2015.

[5] S. Grinstein et al., Module production of the one-arm AFP 3D pixel tracker, JINST 12 (2017) C01086, [1611.01005].

[6] ATLAS Collaboration, J. Lange et al., Beam tests of an integrated prototype of the ATLAS Forward Proton detector, JINST 11 (2016) P09005, [1608.01485].

[7] M. Capeans et al., ATLAS Insertable B-Layer Technical Design Report, Tech. Rep. CERN-LHCC-2010-013. ATLAS-TDR-19, Sep, 2010.

[8] L. Nozka, L. Adamczyk, G. Avoni, A. Brandt, P. Buglewicz, E. Cavallaro et al., Construction of the optical part of a time-of-flight detector prototype for the afp detector, Opt. Express 24 (Nov, 2016) 27951-27960.

[9] https://twiki.cern.ch/twiki/bin/view/AtlasPublic/ForwardDetPublicResults. 